\title{
Digital 3D Ecosystem Modeling Oriented to Machine Vision
}

\author{
Yijie Hao ${ }^{1}$, Wenjing Han $^{2}$, Chunfeng Shen ${ }^{3}$ and Shun Zhang ${ }^{4, *}$ \\ ${ }^{1}$ Rabun Gap-Nacoochee School, Shanghai 200092, China \\ ${ }^{2}$ Environmental Protection Department of Shandong Province, Shandong 250012, China \\ ${ }^{3}$ Shanghai Baosight Software Co., Ltd, Shanghai 200000, China \\ ${ }^{4}$ The CAD Research Center, Tongji University, Shanghai 200092, China \\ *Corresponding author
}

\begin{abstract}
This paper introduces the concept of ecological system to the design of the product, put forward the concept of product digital ecosystem and established the corresponding model, designed to draw lessons from ecological system, and apply the mechanism of self-organization and adaptive, when design demand change, product system can carry on the corresponding structural changes independently, implement the evolutionary design of the product, to meet the needs of people for the product function, improve efficiency, promote the development of product design. This article first has carried on the modeling of product digital ecosystem, defines the factors of the model such as individual, population, community and ecosystem in detail. At the same time, combining with the infectious disease model, the paper puts forward the evolution model of the ecological system complex network, and introduce products ecosystem evolution with specific instances of process. And, the concept of entropy is introduced into the product digital ecosystem, puts forward digital ecosystem evolutionary algorithm based on assembly relation network, implement product evolutionary design process independently. Finally, apply the theory of this article to engine products, and has obtained the expected effect.
\end{abstract}

Keywords-component; product digital ecosystem; assembly relation network; design entropy; digital ecosystem evolution

\section{INTRODUCTION}

Ecosystem is a self-organizing, adaptive systems, when one or more elements is changed, the rest of the system can automatically happen corresponding change, to ensure the smooth running of the whole system. This paper introduces the concept to the product design. Products ecosystem is divided into four levels, ecological system, population, community, individual, form a complex network. Driving force of the production system of evolution is the change of external environment, namely the input of design change information, the evolution of the system process is also the evolution of the complex network. The material carrier of the product system is the structure, the ultimate goal of evolutionary computation is get the optimal solution, make the structure to achieve the optimal state. This paper based on the entropy theory, set the optimization goal of evolutionary computation with the theory of entropy, so as to lay a foundation for design and implementation of the evolutionary algorithm.

\section{The Modeling of Product Digital Ecosystem}

\section{A. The Definition of Product Digital Ecosystem}

Ecosystem is a self-organizing, adaptive system, when one or more elements is changed, the rest of the system can automatically happen corresponding change, to ensure the smooth running of the whole system. This paper introduced the concept of the ecological system to the product design process, thus puts forward the concept of product digital ecosystem. In this article, the product digital ecosystem is defined as follows: in a certain space, different elements constitute a unified whole, through the exchange and transfer of substance, energy, information to effect each other, can achieve a certain function, and can adapt to the environment, the complete function unit called product digital ecosystem. In this paper, the engine as an instance of product digital ecosystem, specific introduce the product modeling process and the applications of the digital ecosystem.

\section{B. Product Digital Ecosystem Components Modeling}

In the product digital ecosystem, we regard the smallest unit of products, parts as individuals in the ecosystem. For example, the piston, connecting rod, connecting rod, crankshaft of crank connecting rod mechanism. In CSG model, entity generated by the basic geometric elements through a variety of operations, in this paper, regard the characteristics of parts as the most basic component unit, through feature tree to produce the final parts.

1) Mathematical formula to describe individual

$\mathrm{PI}=\{$ Feature ,FeatureOrder ,FeatureRelation $\}$

$$
\text { Feature }=\left\{\mathrm{F} \_1, \mathrm{~F} \_2, \mathrm{~F} \_3, \cdots, \mathrm{F} \_\mathrm{n}\right\}
$$

FeatureOrder $=\left\{\mathrm{F} \_1 \rightarrow \mathrm{F} \_2 \rightarrow \mathrm{F} \_3 \rightarrow \cdots \rightarrow \mathrm{F} \_\mathrm{n}\right\}$

\section{FeatureRelation $=\mathrm{A}$}

Among them, PI represent the individual, Feature represent the set of feature, FeatureOrder represent of the order of features, FeatureRelation represent the relationship of feature, use relationship matrix A to describe, $F_{-} \mathrm{i}(\mathrm{i}=1,2, \cdots, \mathrm{n})$ represent specific feature. 


\section{2) The elements of individual}

Individual components mainly include the various characteristics of CAD system, such as convex platform, grooves, rotator, rotating groove, hole, rib, notching, strengthening rib, entity, mixing, many section entities, has removed many section entities, etc. Their descriptions are as follows.

convex platform: the main parameters, such as size, until the next, until the plane etc. Outline, direction, etc;

groove: the main parameters, such as size, until the next, until the plane etc. Outline, direction, etc.;

$$
\mathrm{F}=\left\{\text { Type }, \mathrm{p} \_1, \mathrm{p} \_2, \cdots, \mathrm{p} \_n\right\}
$$

Among them, $\mathrm{F}$ represent feature, Type represent the kind of feature, $P \_i(i=1,2, \cdots, n)$ represent the parameter of feature.

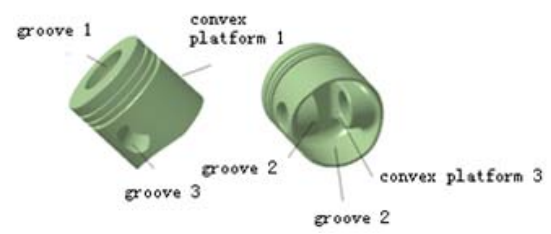

FIGURE I. THE CHARACTERISTICS MAKE UP THE PARTS WITH ASPECIFIC ORDER

In this paper, the characteristics make up the parts with a specific order, the space position of two features is described by spatial predicate logic network.

The generation relationship between characteristics can also use relation matrix to describe:

$$
A=\left[\begin{array}{cccc}
a_{11} & a_{12} & \ldots & a_{1 n} \\
\alpha_{21} & \alpha_{22} & \ldots & a_{2 n} \\
\vdots & \vdots & \vdots & \vdots \\
\alpha_{m 1} & a_{m 2} & \ldots & a_{m n}
\end{array}\right]
$$

In this paper, a_ij( $i=1,2, \cdots, n ; j=1,2, \cdots, n)$ represent the relationship between feature $I$ and $j$. When a_ij=k,k $\in$ $\{1,2,3,4,5,6,7,8\}$, state that feature I and $\mathrm{j}$ has neighboring relations, and I generate before $\mathrm{j}$, feature $\mathrm{I}$ and $\mathrm{j}$ has the spatial predicate logic relationship described by $\mathrm{k}$. When $\mathrm{a} \_\mathrm{ij}=-\mathrm{k}, \mathrm{k} \in$ $\{1,2,3,4,5,6,7,8\}$, state that feature $\mathrm{I}$ and $\mathrm{j}$ has neighboring relations, and $\mathrm{j}$ generate before $\mathrm{i}$, feature $\mathrm{I}$ and $\mathrm{j}$ has the spatial predicate logic relationship described by $\mathrm{k}$ 。 Other condition, a_ij $=0$.

\section{Product Population}

\section{1) The definition of population}

Population, that is, "certain parts:" a collection of the same kind products individuals in a certain space, have the same structure. Such as, connecting rod population, piston population, and the crankshaft connecting rod cover population, etc. As shown in figure 2.6, the piston population has four individuals, connecting rod population has four individuals, connecting rod cover population has four individuals, only an individual crankshaft populations. A mathematical formula description of the population.

$\mathrm{PP}=\{$ IndividualType ,scale ,PopulationRelation ,Community T ype

Among them, PP represent population, IndividualType represent individual kind of population, scale represent the scale of population, PopulationRelation represent the relationship of individuals, CommunityType represent the community.

2) The matrix description of the relationship between population

\section{a) Assembly relation}

The relationship between the different population mainly reflect by the relationship between individuals within the population and other populations. Among product digital ecosystem, the relationship between the population is mainly for assembly relation, including consistency, contact constraint, offset constraints, angle and solid coupling constraints, etc.

b) The matrix description of assembly relation

If a system has $\mathrm{n}$ individuals from different populations, the matrix to describe the relationship between the populations can use the following:

$$
\mathrm{B}=\left[\begin{array}{cccc}
b_{11} & b_{12} & \ldots & b_{1 \mathrm{n}} \\
b_{21} & b_{22} & \ldots & b_{2 n} \\
\vdots & \vdots & \vdots & \vdots \\
b_{n 1} & b_{n 2} & \ldots & b_{n n}
\end{array}\right]
$$

Among them, ( $i=1,2, \ldots, n ; j=1,2, \ldots, n$ ) represent the relationship between individual $\mathrm{I}$ and $\mathrm{j}$, is the set of simple assembly relation.

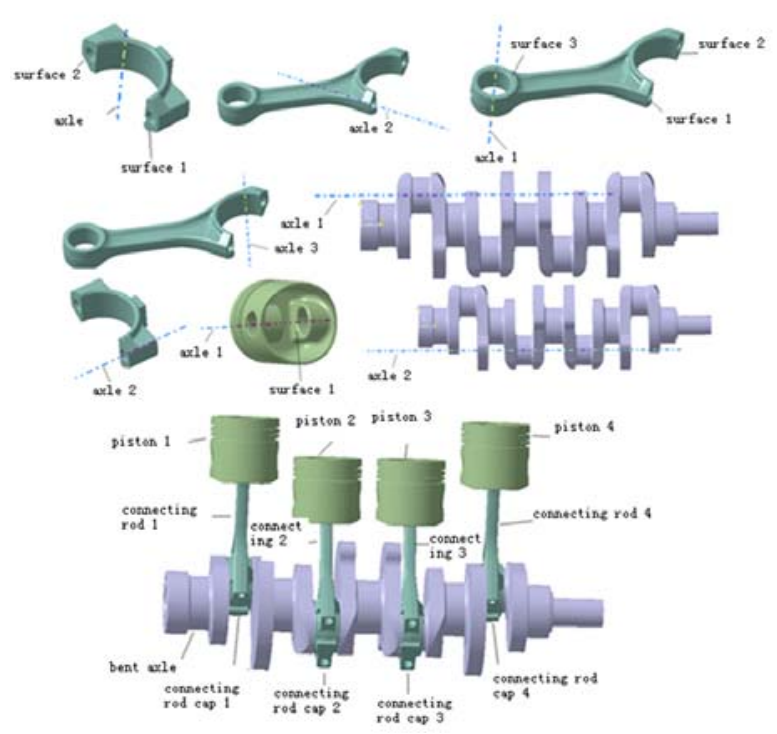

FIGURE II. THE ORDER OF THE INDIVIDUAL AS FOLLOWS

As shown in figure 2, population connecting rod, piston, connecting rod cap population and the crankshaft through the 
relationship between each other make up the collection. The order of the individual as follows: piston 1, piston 2, piston 3, piston 4 and connecting rod 1, connecting rod 2, connecting rod 3 ,connecting rod 4 , connecting rod cover 1 , connecting rod cover 2, connecting rod cover 3 , connecting rod cover 4, crankshaft. The relationship between the set can be expressed in the following matrix.

$$
\mathbf{B}=\left[\begin{array}{ccccccccccccc}
0 & 0 & 0 & 0 & (4,8) & 0 & 0 & 0 & 0 & 0 & 0 & 0 & 0 \\
0 & 0 & 0 & 0 & 0 & (4,8) & 0 & 0 & 0 & 0 & 0 & 0 & 0 \\
0 & 0 & 0 & 0 & 0 & 0 & (4,8) & 0 & 0 & 0 & 0 & 0 & 0 \\
0 & 0 & 0 & 0 & 0 & 0 & 0 & (1,8) & 0 & 0 & 0 & 0 & 0 \\
(4,8) & 0 & 0 & 0 & 0 & 0 & 0 & 0 & (6,4,4) & 0 & 0 & 0 & 4 \\
0 & (4,8) & 0 & 0 & 0 & 0 & 0 & 0 & 0 & (6,4,4) & 0 & 0 & 4 \\
0 & 0 & (4,8) & 0 & 0 & 0 & 0 & 0 & 0 & 0 & (6,4,4) & 0 & 4 \\
0 & 0 & 0 & (4,8) & 0 & 0 & 0 & 0 & 0 & 0 & 0 & (6,4,4) & 4 \\
0 & 0 & 0 & 0 & (6,4,4) & 0 & 0 & 0 & 0 & 0 & 0 & 0 & 4 \\
0 & 0 & 0 & 0 & 0 & (6,4,4) & 0 & 0 & 0 & 0 & 0 & 0 & 4 \\
0 & 0 & 0 & 0 & 0 & 0 & (6,4,4) & 0 & 0 & 0 & 0 & 0 & 4 \\
0 & 0 & 0 & 0 & 0 & 0 & 0 & (6,4,4) & 0 & 0 & 0 & 0 & 4 \\
0 & 0 & 0 & 0 & 4 & 4 & 4 & 4 & 4 & 4 & 4 & 4 & 0
\end{array}\right]
$$

The number in Assembly relation matrix represent specific assembly relation, details are shown in table 2.2, when there are multiple relationship between the two parts, use multiple group. Among them, 0 means no direct relationship between each other.

\section{3) The network description of assembly relation}

The relationship between populations can be described by assembly relation network $\mathrm{G}=(\mathrm{V}, \mathrm{E}), \mathrm{V}$ represent the set of individuals, E represent the set of relationship.

$$
\left\{\begin{array}{l}
G-(V, E) \\
W(e) \in\{a\}
\end{array}\right.
$$

Assembly relation network $G$ is a collection of the set of nodes and the set of edges between nodes, two individuals have the assembly relation if two nodes linked by solid lines, the values of the edge is the subset of the set of encoding of the assembly relations.

The nodes in the graph is individuals of product digital ecosystem, different colors represent different populations individuals. Among them, the dashed lines represent the relationship between the individual within the same species, mainly competition relations; The solid line shows the relationship between the individual of different populations, as a collection of assembly relation, the number represent a particular assembly relations, specific meaning can be found in table 2.2. When the assembly relation between the two individuals is not the only, described in the form of a diverse group.

\section{Digital ECOSYSTEM EVOLUTIONARY AlgORITHM BASED ON ASSEMBLY RELATION NETWORK}

In view of the design change, in this paper, the ideas of system evolution as follows: at first, pass change to the directly related individual, individual generate contradiction with environment, individual evolution so as to adapt to environmental changes. Change in the individual transfer by assembling relation network, leading to changes of the environment of other individuals in the related, single or multiple individual cooperative coevolution, reduce the contradiction between the individual and the environment, to adapt to the environment changes.

\section{A. The Definition of Product Gene}

The relationship between the different population mainly reflect by the relationship between individuals within the population and other populations. Among product digital ecosystem, the relationship between the population is mainly for assembly relation, including consistency, contact constraint, offset constraints, angle and solid coupling constraints, etc.

\section{B. Assembly Relation Network}

The population set shown in figure 3 can be represent with the following assembly relation network.

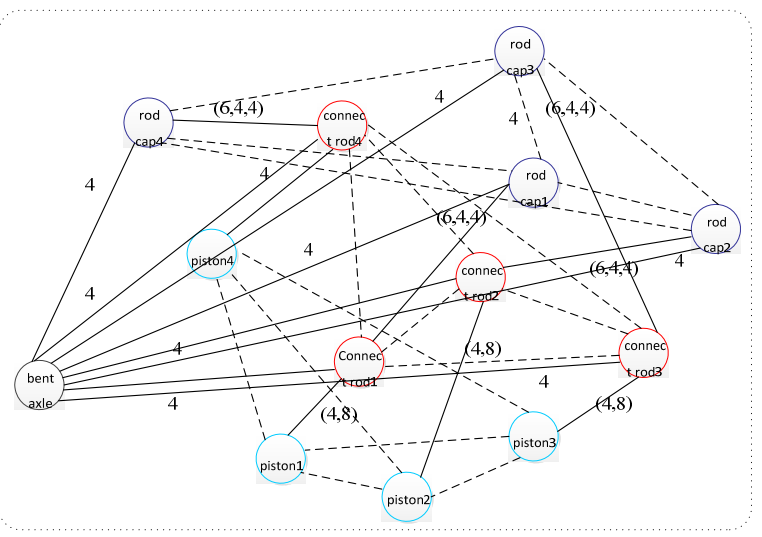

FIGURE III. THE NETWORK OF ASSEMBLY RELATION OF CRANKLINK MECHANISM

Assembly relation network $G$ is a collection of the set of nodes and the set of edges between nodes, two individuals have the assembly relation if two nodes linked by solid lines, the values of the edge is the subset of the set of encoding of the assembly relations.

The nodes in the graph is individuals of product digital ecosystem, different colors represent different populations individuals. Among them, the dashed lines represent the relationship between the individual within the same species, mainly competition relations; The solid line shows the relationship between the individual of different populations, as a collection of assembly relation, the number represent a particular assembly relations, specific meaning can be found in table 2.2. When the assembly relation between the two individuals is not the only, described in the form of a diverse group.

\section{CONCLUSION}

This paper introduces the concept of ecological system to the design of the product, put forward the concept of product digital ecosystem and established the corresponding model, designed to draw lessons from ecological system, and apply the mechanism of self-organization and adaptive, when design demand change, product system can carry on the corresponding structural changes independently, implement the evolutionary design of the product, to meet the needs of people for the product function, improve efficiency, promote the development of product design. This article first has carried on the modeling 
of product digital ecosystem, defines the factors of the model such as individual, population, community and ecosystem in detail. At the same time, combining with the infectious disease model, the paper puts forward the evolution model of the ecological system complex network, and introduce products ecosystem evolution with specific instances of process. And, the concept of entropy is introduced into the product digital ecosystem, puts forward digital ecosystem evolutionary algorithm based on assembly relation network, implement product evolutionary design process independently. Finally, apply the theory of this article to engine products, and has obtained the expected effect.

\section{ACKNOWLEDGMENT}

The research work presented in this paper was supported by National Science and Technology Support Program: Electronic Devices Assembly High-speed Precision Robot and Automation Production Line R \& D and Industrialization (2015BAF10B01); Shanghai Science and Technology Commission Project: Multi-information visual recognition and 3D reconstruction key technology for industrial large-scale mechanical intelligent network control (14JC1402203).

\section{REFERENCES}

[1] Hobday, Mike. Product complexity, innovation and industrial organization[J]. Research Policy.1998, 26(6): 689-710.

[2] Hansen, K.L. and H. Rush. Hotspots in complex product systems: Emerging issues in innovation management[J]. Tec novation. 1998,18(89): 555-561.

[3] Davies, A. and T. Brady. Policies for a complex product system[J]. Futures. 1998,30(4): 293-304.

[4] Claudia Eckert, P.J. Clarkson, W. Zanker. Change and customization in complex engineering domains[J].Research in Engineering Design,2004,15:1-21. .

[5] Kamel Rouibaha, K.R. Caskeyb. Change management in concurrent engineering from a parameter perspective[J].Computers in Industry,1997,2 (50):15-34. .

[6] E. Frike, B. Gebard, H. Negele. Coping with Changes-Causes, findings and strategies[J]. Systems Engineering, 2000,3(4):321-333.

[7] Randal J. Peters, Ken Barker. Change Propagation in an Axiomatic Model of Schema Evolution for Object based Management Systems[J].Lecture Notes in Computer Science,2006, 2065: 142-162.

[8] T. Jarratt, C. Eckert, P.J. Clarkson. Change practice during complex product design[C].14th CIRP Design Seminar. Cairo, Egypt, 2004.

[9] Pierre Baldi, Soren Brunak. Bioinformatics- the machine learning approach[M].2001:1-370.

[10] Conrad, M., Pattee, H. H. Evolution Experiments with an Artificial Ecosystem[J]. Journal of Theoretical Biology. 1970, 3(28): 393-409. 